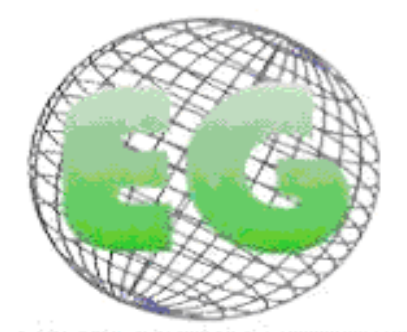

ISSN 1695-6141 $N^{\circ} 26$

www.um.es/egloball

\title{
CLÍNICA
}

\section{Estudio cuasi-experimental sobre las terapias de relajación en pacientes con ansiedad}

Quasi-experimental relaxation therapies in patients with anxiety

\section{*Soriano González, Javier}

\author{
*Enfermero. Servicio Murciano de Salud. Murcia. E-mail javo_8888@hotmail.com \\ Palabras clave: ansiedad; terapias de relajación; efectividad
}

Keywords: anxiety; relaxation therapy; effectiveness.

\section{RESUMEN}

Introducción: Los trastornos de ansiedad son muy frecuentes en atención primaria y especializada. Para reducir la ansiedad se recomienda el uso de terapias de relajación como terapia alternativa a la psicofarmacología. El objetivo del estudio será averiguar si las terapias de relajación benefician a los pacientes con ansiedad.

Material y métodos: Se utilizó metodología cuantitativa: estudio cuasi-experimental de antes y después, longitudinal, prospectivo. Sujetos a estudio: pacientes diagnosticados de ansiedad y que acepten participar en la terapia de relajación en el centro de salud mental Infante (Murcia), desde diciembre hasta marzo de 2010. $(n=39)$. Variable independiente: terapia de relajación; variable dependiente: ansiedad. Otras variables: sexo, edad, nivel educativo y tratamiento con psicofármacos. Se utiliza la escala validada: STAl; se comparan puntuaciones pre-post-intervención (se aplica t de student, xi cuadrado (intervalo de confianza $p<0$ 05).

Resultados: Realizan el test STAI pre-post 39 pacientes, 69\% mujeres y 31\% hombres. La edad media: $39{ }^{\prime} 84$ años. Un $77 \%$ tomaban medicación y el $23 \%$ no. Predominaba claramente el de educación secundaria obligatoria (ESO).

La media de ansiedad estado antes fue de 68 y después de 56. STAl estado (antes/después) con un nivel de significación de $p<0$ 027, por lo que se aceptó la hipótesis nula. Se observó que en los pacientes que no tomaban psicofármacos, el descenso de ansiedad era significativo con una $p<0$ '036.

Conclusiones: Los talleres de técnicas de relajación son útiles en la reducción de la ansiedad en atención especializada, además son más eficaces en pacientes con ansiedad que no tomen medicación. 


\section{ABSTRACT}

Introduction: Anxiety disorders are highly prevalent in primary and specialty care. To reduce anxiety the use of relaxation therapy is recommended as an alternative therapy to Psychopharmacology. The aim of the study is determine whether relaxation therapies benefit patients with anxiety.

Materials and Methods: We used quantitative methodology: a quasi-experimental study before and after a, longitudinal, prospective study. Subjects studied: patients diagnosed with anxiety who agreed to participate in relaxation therapy at the mental health center Infante (Murcia), from December 2009 to March 2010 . $(\mathrm{N}=39)$. Independent variable was relaxation therapy and the dependent variable was anxiety. Other variables were sex, age, educational level and treatment with psychotropic drugs. Validated scale used was STAI, scores comparing pre-post-intervention (t student applies, xi square confidence interval $(p<0.05)$.

Results: The test was performed by pre-post STAI 39 patients, $69 \%$ female and $31 \%$ male. The mean age was $39 ' 84$ years. $77 \%$ were taking medication and $23 \%$ were not. There was a clearly dominance of secondary education (ESO).

Mean state anxiety before was 68 and after 56 . STAl state (before / after) with a significance level of $p<0^{\prime} 027$, so the null hypothesis was accepted. It was observed that in patients not taking psychotropic drugs, the decrease in anxiety was significant at $p<0^{\prime} 036$.

Conclusions: Workshops in relaxation techniques are helpful in reducing anxiety in specialized care, and are more effective in patients with anxiety who do not take medication.

\section{INTRODUCCIÓN}

Según la Organización Mundial de la Salud (OMS), los trastornos mentales de ansiedad y depresión se encuentran entre los trastornos psiquiátricos de mayor prevalencia ${ }^{1-2}$, siendo la ansiedad la segunda causa más frecuente de diagnósticos psiquiátricos emitidos en atención primaria ${ }^{3}$. Estos trastornos constituyen el principal motivo de consulta en lo que a trastornos mentales se refiere, tanto en atención primaria como en especializada, relacionándose con una gran cantidad de demandas que refieren dolencias somáticas inespecíficas que no corresponden con patologías concretas ${ }^{4-5}$.

Se estima que un $22 \%$ de la población sufre episodios de ansiedad ${ }^{6}$; según la encuesta National Comorbidity Survey, una de cada cuatro personas presenta síntomas de un trastorno de ansiedad en algún momento de su vida, existiendo una prevalencia del doble en las mujeres que en los hombres y además los trastornos de ansiedad también muestran una prevalencia elevada en ancianos y en niños. Los trastornos de ansiedad con frecuencia se asocian a síntomas depresivos ${ }^{7}$.

La NANDA define ansiedad como "vaga sensación de malestar o amenaza acompañada de una respuesta autonómica" ${ }^{\prime \prime}$ la ansiedad produce cambios psicológicos, fisiológicos y conductuales. Psicológicamente se manifiesta como una sensación compleja y desagradable que abarca desde la inquietud hasta el pánico, miedo a morir, volverse loco o a perder el control, preocupación, sensación de estar en peligro, sensación de indefensión, etc. Fisiológicamente la ansiedad se expresa a través del sistema nervioso autónomo, aumentando la frecuencia cardiaca (FC), respiratoria (FR), elevando la tensión arterial (TA), sudoración, aumento de lactato en sangre, temblores, hormigueo en brazos y piernas, sequedad de la boca, cansancio, necesidad de orinar con mayor frecuencia, sudoración, alteraciones digestivas (náuseas, vómitos), dificultad para respirar, etc. Por ultimo, afecta a la conducta de manera que hay disminución del rendimiento, de la concentración, problemas para divertirse y disfrutar; además existe inquietud e impaciencia y se evitan situaciones en las que se piense que la ansiedad se puede desencadenar. Estos tres componentes 
(fisiológico, psicológico y conductual) o formas de manifestarse la ansiedad, varían según la persona que la padece o incluso en la misma persona pueden aparecer manifestaciones en diferentes momentos. Predominan las manifestaciones físicas, que llegan a pensar que padece una enfermedad grave ${ }^{9}$.

Se conoce que un cierto nivel de ansiedad es beneficioso para proteger a las personas en situaciones de peligro, pero un exceso de esta se transforma en negativo y contraproducente para las personas ${ }^{6}$, produciendo como características mas habituales: temor extremo en situaciones en las que no existe ningún peligro real, dificultades emocionales que interfieren con la vida cotidiana, evitación de situaciones que causan ansiedad ${ }^{7}$.

Spielberger estableció la distinción entre dos tipos de ansiedad:

La ansiedad rasgo: se define como la predisposición personal a sentirse ansioso en situaciones muy diversas.

La ansiedad estado: se define como la respuesta de ansiedad ante estímulos amenazantes $^{10}$.

La pregunta más frecuente entre los pacientes diagnosticados de ansiedad es si la ansiedad puede curarse. Hoy día los trastornos por ansiedad si se diagnostican correctamente y se tratan de una forma apropiada pueden controlarse y, en algunos casos, resolverse de forma definitiva. De todos modos, la respuesta al tratamiento y la evolución depende del tipo de trastorno y del control de los factores de riesgo. Así, las formas agudas de ansiedad, incluyendo el trastorno por angustia, pueden tener una evolución excelente, mientras que el trastorno obsesivo compulsivo y el trastorno por estrés postraumático son más difíciles de tratar y, a menudo, requieren una terapia más prolongada ${ }^{11}$.

Un buen manejo del estrés, una personalidad estable y contar con apoyo social ayudan a prevenir los trastornos por ansiedad.

El tratamiento más utilizado para disminuir la ansiedad es el farmacológico, pero actualmente se emplea un uso excesivo de ansiolíticos como son las benzodiacepinas como el alprazolam y el clonazepan, que proporcionan un rápido alivio de la sintomatología. En España el que más recetan los facultativos es alprazolam en dosis de 0,5 mg, administrado por vía sublingual u oral, y que se puede repetir a los 20 minutos, es una buena alternativa, también el diacepam 5-10 mg, cloracepato dipotásico 5-15 mg o lorazepam 1-2 mg. Por vía oral todas las benzodiacepinas se absorben con facilidad. La absorción por vía intramuscular y rectal es errática, por lo que deben ser evitadas estas vías. Con la vía intravenosa hay que tener precauciones por el riesgo potencial de parada respiratoria, por ello hay que disponer de medidas de reanimación incluyendo flumanezilo, pero el uso de estos psicofármacos tienen el inconveniente de producir efectos adversos como son: ataxia, hipotonía muscular, somnolencia, depresión respiratoria, etc., y a largo plazo producen efectos tales como: tolerancia, abusos, síndrome de abstinencia y dependencia ${ }^{12-13}$.

Los efectos secundarios son la razón por la que los profesionales sanitarios y entre estos el enfermero deben utilizar otras medidas para disminuir la ansiedad que no produzcan sintomatologías adversas ${ }^{3}$; entre otras técnicas para disminuir la ansiedad la más importante en cuanto a validez es la relajación ya que no produce efectos adversos y disminuye la ansiedad. 
"Se considera interesante el aprendizaje de técnicas de relajación en la formación de futuros profesionales de Enfermería porque actuando desde lo físico, que sería la relajación muscular, se actúa en el plano psíquico, encontrando el paciente grandes beneficios" ${ }^{14}$, por ello se llega a la conclusión de que las técnicas de relajación reducen los niveles de activación autonómica y la experiencia desagradable de ansiedad, mejora el sueño y otros síntomas asociados al estrés, así como aumenta la motivación a continuar el tratamiento si se practica regularmente ${ }^{4}$.

Según la teoría del autocuidado de Dorotea Orem: "El autocuidado es una actividad aprendida por los individuos, orientada hacia un objetivo. Es una conducta que existe en situaciones concretas de la vida, dirigida por las personas sobre sí mismas, hacia los demás o hacia el entorno, para regular los factores que afectan a su propio desarrollo $y$ funcionamiento en beneficio de su vida, salud o bienestar"; así pues se entiende que la relajación formaría parte de un beneficio para la vida, la salud y / o el bienestar ${ }^{15-16}$.

En la teoría de la enfermería psicodinámica, de Hildegard Peplau, la enfermería es un importante proceso interpersonal y terapéutico. Funciona en términos de cooperación con otros procesos humanos que hacen de la salud una posibilidad para los individuos en las comunidades, lo que significa que es importante que el profesional de enfermería coopere en la mejora de la salud en pacientes con ansiedad y en pacientes en general ${ }^{17}$.

Burgo Varo, ML et alt en su estudio: Intervención grupal en los trastornos de ansiedad en atención primaria: técnicas de relajación y cognitivo-conductuales, llegaron a la conclusión de que la ansiedad disminuía considerablemente tras la intervención ${ }^{4}$.

En distintos estudios se llegó a la conclusión de que "el entrenamiento en relajación es uno de los procedimientos más utilizados para disminuir la sintomatología de la ansiedad"4,18-

Por tanto, la solución más efectiva para disminuir la ansiedad es la relajación. Tras esto se considera que los talleres centrados en la aplicación de la terapia de relajación son una herramienta de autoayuda en el afrontamiento individual y familiar de personas con ansiedad. Actualmente se están realizando talleres de relajación para enseñar y demostrar a las personas con ansiedad que se puede disminuir a través de la relajación ${ }^{19}$.

Además de la relajación existen una serie de recomendaciones que disminuyen la ansiedad, como son 20-21: Mantener una alimentación sana y saludable: dietas con poca sal; reducir el consumo de café, tabaco, alcohol; tener una dieta equilibrada (55\% hidratos de carbono, $30 \%$ lípidos, $15 \%$ proteínas); evitar alimentos con aditivos; ingesta reducida de colesterol; beber aproximadamente dos litros diarios de agua; comer de manera regular, evitando los atracones; ingesta adecuada de vitaminas y minerales y consumir una adecuada cantidad de fibra; realizar actividad física que no sea muy intensa y que sea eficaz, divertida y constante; aprender a decir que no, porque no se puede ir sobrecargado, y lo más importante es la salud de uno mismo; delegar en otros parte de nuestras responsabilidades: hay que aceptar que si delegas la persona lo hará de forma diferente a la de uno mismo pero que no lo hará peor; organizar el tiempo, dando prioridad a lo más importante.

En el protocolo de relajación que se utiliza en los centros de salud mental del servicio murciano de salud, se escoge como principal método de relajación, la técnica de relajación de Jacobson y el entrenamiento en respiración. Se estudiaron varios meta-análisis ${ }^{22-26}$, entre ellos uno de la facultad de Psicología de Murcia, los cuales concluían en que tanto la relajación muscular, como el entrenamiento autógeno obtienen resultados parecidos de 
diversas dolencias, por lo que se considera una técnica útil en Atención Primaria y especializada.

Por tanto se llega a la conclusión de que la relajación muscular es una estrategia útil tanto para trastornos psíquicos, como para otras enfermedades físicas ${ }^{21}$.

El fundamento de la relajación progresiva de Jacobson se basa en que si somos capaces de identificar los músculos de nuestro cuerpo, tensarlos y luego soltarlos, nos sentiremos relajados y podremos controlar la ansiedad.

La relajación de Jacobson nos permite aprender a tensar y después soltar o relajar de manera secuencial varios grupos de músculos a la vez, de forma que el paciente tendrá que prestar atención a las sensaciones asociadas a la tensión y relajación de los músculos, de manera que lo que al final consiga es ser capaz de reducir la tensión muscular cuando uno quiera hacerlo en la vida cotidiana ${ }^{21}$.

Con la práctica de la relajación de Jacobson se pretenden alcanzar una serie de objetivos, como son: aliviar o eliminar la tensión muscular, favorecer el descanso y el sueño, disminuir la ansiedad y conseguir disminuir el dolor y la hipertensión.

En definitiva, el propósito final de la relajación de Jacobson es enseñar al paciente la técnica de autocontrol ante cualquier situación ansiógena ${ }^{21}$.

Por tanto, la hipótesis que nos planteamos para este estudio es que las terapias de relajación benefician a los pacientes con ansiedad.

El objetivo principal será:

Averiguar si las terapias de relajación benefician a los pacientes con ansiedad.

Los objetivos secundarios serán:

1) Valorar el grado de ansiedad antes de realizar la terapia de relajación.

2) Realizar la terapia de relajación.

3) Observar si el grado de relajación ha variado con respecto al primer objetivo secundario.

\section{MATERIAL Y MÉTODOS}

Se utilizó metodología cuantitativa, siendo un estudio cuasi-experimental de antes y después, longitudinal, prospectivo. Se realizó un estudio cuasi-experimental (antes y después) porque se realizaron dos mediciones una previa a la intervención y otra posterior, y además, en el estudio no se utilizó un grupo control para valorar el nivel de ansiedad de los pacientes; longitudinal, pues se hicieron dos mediciones en dos momentos diferentes, y prospectivo, porque aún no se habían medido los resultados.

El estudio se llevó a cabo en el centro de salud mental Murcia Infante durante los meses de diciembre de 2009, hasta marzo de 2010.

La población diana se definió como todos aquellos pacientes diagnosticados de ansiedad de la zona básica de salud Murcia Infante. $\mathrm{N}=70$. 
Los criterios de inclusión que se utilizaron fueron: pacientes que aceptaran participar en el estudio y recibir la terapia.

Los criterios de exclusión: aquellos pacientes que no aceptaran participar en el estudio y recibir la terapia.

El tamaño de la muestra con la técnica del calculo muestral para poblaciones finitas fue de $\mathrm{n}=39$ personas y se utilizó como técnica de muestreo, el muestreo no probabilístico por conveniencia.

La variable independiente elegida fue la terapia de relajación y se definió como una variable cualitativa nominal.

La variable dependiente fue la ansiedad como variable cuantitativa discreta.

Los instrumentos de recolección de datos que se emplearon para el estudio fueron el STAI, el programa Microsoft Excell para recogida de gráficos y el programa informatico SPSS versión 2009.

EI STAI permite la evaluación de la ansiedad como estado transitorio (Ansiedad / Estado) y como rasgo latente (Ansiedad / Rasgo). Esta prueba se puede utilizar tanto en adolescentes, como en adultos. Consta de dos partes, la primera parte (Ansiedad / Estado) evalúa un estado emocional transitorio, caracterizado por sentimientos subjetivos, conscientemente percibidos, de atención y aprensión y por la hiperactividad del sistema nervioso autónomo. La segunda (Ansiedad / Rasgo) señala una propensión ansiosa, relativamente estable, que caracteriza a los individuos con tendencia a percibir las situaciones como amenazadoras.

La escala Ansiedad-Rasgo, consta de veinte afirmaciones en las que se pide a los sujetos describir cómo se sienten generalmente. La escala Ansiedad-Estado también consiste en veinte afirmaciones, pero las instrucciones requieren que los sujetos indiquen cómo se sienten en un momento dado.

La escala Ansiedad-Rasgo puede ser utilizada para determinar los sujetos que varíen en su disposición a responder a la tensión psicológica con niveles de ansiedad distintos AnsiedadEstado. Esta escala sirve también para establecer los niveles de intensidad Ansiedad-Estado inducidos por procedimientos experimentales o en situaciones de campo, en un momento dado o a través de un continuo. Las puntuaciones para ambas escalas varían desde una puntuación mínima de 20 a una máxima de 80 en progresión con el nivel de ansiedad. Los sujetos contestan a cada una de las afirmaciones ubicándose en una escala de cuatro dimensiones que van de 1 a 4 . En la escala Ansiedad-Estado son: 1-No en absoluto, 2-Un poco, 3-Bastante, 4-Mucho, y en la escala Ansiedad-Rasgo son: 1-Casi nunca, 2- Algunas veces, 3- Frecuentemente, 4-Casi siempre, con una confiabilidad entre el 0.73 y 0.86 , siendo pues una escala validada.

Además se utilizará el programa informático Microsoft Excel para la recogida de datos.

La terapia de relajación no la realizó el investigador, la realizaron las enfermeras especializadas en salud mental del C.S.M (centro de salud mental) del Infante (Murcia).

El procedimiento fue el siguiente: primero se realizaron dos grupos compuestos por un total de 20 personas cada uno, uno comenzaría el 1 de diciembre de 2009 y finalizaría a 
mediados de marzo de 2010 y otro comenzaría a mediados de diciembre de 2009 y finalizaría a finales de marzo de 2010.

En los dos grupos se realizó el mismo procedimiento estando por tanto en igualdad de condiciones. Cada grupo de 20 personas realizó 8 sesiones de relajación, según el modelo de Jacobson (relajación con tensión).

Antes de comenzar la primera sesión se les dio a los pacientes el consentimiento informado para que lo rellenaran y una fotocopia del STAI. Se realizaron un total de 8 sesiones en las que la sala cumplía con las siguientes condiciones: estar insonorizada o al menos lo más silenciosa posible, la temperatura era agradable (ni muy fría ni muy calurosa) La luz era tenue (en las primeras sesiones se practicó con la luz encendida para que los pacientes pudieran seguir las instrucciones del terapeuta, una vez que las automatizaron disminuyó la intensidad), el asiento era cómodo para que el paciente estuviera lo más a gusto posible; la vestimenta del paciente debía ser cómoda (se recomendó evitar utilizar cualquier cosa que apretara, ya sea cinturón, etc. o que moleste), no permanecer con objetos en el regazo, si hubiera alguien que le molestan las gafas se recomendó que se las quitara, fue fundamental la desconexión de los teléfonos móviles.

Durante las 8 sesiones la posición era sentado en la silla con la espalda bien apoyada en el respaldo, la cabeza recta, evitando que se inclinaran a algún lado, los pies bien apoyados en el suelo, sin cruzar las piernas y las manos sobre los muslos.

En la primera sesión se demostró cómo se tensan y se relajan los grupos musculares que posteriormente intervendrían. La tensión duraba un tiempo corto (de 3-5 segundos). Cuando se relajaban los músculos se hacía de golpe dejándolos lo más sueltos y flojos posibles durante un tiempo mucho más largo (15 segundos).

En todas y cada una de las sesiones era importantísima la voz del terapeuta, ya que tenia que aumentar de volumen en el momento de la tensión y disminuir el volumen en el momento de la relajación; era imprescindible que se notara la diferencia de volumen entre la relajación y el momento de tensión.

Al comenzar y finalizar todas y cada una de las ocho sesiones, se realizaban alrededor de cinco respiraciones diafragmáticas, que consisten en una inhalación y una exhalación que dura el doble de tiempo que la inhalación. Tras realizar las cinco respiraciones diafragmáticas se comenzaba con la tensión- relajación muscular. En primer lugar se tensaban los brazos (izquierdo y derecho) de modo que se cerraba el puño y se estiraba el brazo lo máximo posible para relajarlo dejándolo caer lo más suelto posible a la vez que se centraban en las sensaciones de relajación que producían (esto se hacía tras cada tensión); después se tensaban los hombros y espalda, de forma que el paciente se incorporaba en la silla y llevaba los hombros hacía detrás intentando juntar las escápulas, luego volvían a la posición de relajación dejando los hombros caídos y la espalda floja.

Posteriormente se tensaba el tórax de manera que se hacía una inspiración profunda y se retenía el aire, mientras que se observaba la tensión en los músculos del tórax, se expulsaba el aire poco a poco y se iba viendo la diferencia entre la tensión y la relajación, siendo conscientes de las sensaciones de relajación que se experimentaban; el siguiente paso sería tensar los músculos del abdomen de forma que el estómago quedaba duro, apretando hacía adentro, para relajarlo dejaban hinchado el abdomen; la siguiente tensión fue en las piernas y pies (derecho e izquierdo) haciendo tensión de manera que los pies quedaran hacia adentro, levantando la pierna, estirándola al máximo; para relajarla dejaríamos caer la pierna 
de golpe y seguiríamos centrados viendo las sensaciones asociadas a la relajación de cada una de las partes del cuerpo correspondientes. En penúltimo lugar se tensaban el cuello de forma que se empujaba la barbilla hacia abajo evitando tocar el pecho, para relajar se dejaba el cuello lo más suelto y flojo posible volviéndolo a su posición inicial; por último, se tensaba la cabeza que se dividiría en tres partes: frente (arrugando la frente y volviéndola a su posición inicial para relajarlas), ojos , nariz y parte superior de las mejillas (cerrando los ojos fuertemente y arrugando la nariz) y finalmente la mandíbula (apretando los dientes fuertemente y para relajarla se volvía a la posición inicial) y para concluir se hicieron otras cinco respiraciones diafragmáticas.

Estos ejercicios se realizaron de la misma manera en todas y cada una de las 8 sesiones para ambos grupos de 20 personas.

Tras finalizar la octava sesión se les dio una segunda fotocopia del STAI para que la rellenaran y la entregaran para ver si habían cambios en la ansiedad tras la terapia. Una vez recogidas ambos STAI de cada uno de los pacientes se compararon (antes y después) y se valoró si existían variaciones o no del nivel de ansiedad de cada paciente.

Los recursos materiales utilizados fueron: fotocopias (una para cada paciente) del STAI, un CD de música de relajación (con contenido de canciones de musica clásica y relajante, sonidos del mar, etc.) para crear un ambiente tranquilo y relajante, una sala de exposiciones (la cual estará aislada, de modo que no moleste ningún ruido exterior a dicha sala), un equipo de música reproductor de mp3 ,sillas para todos los pacientes, un ordenador y los programas informáticos necesarios para el estudio.

En el estudio se utilizó la Chi cuadrado para comparar las variables y la t de student para ver las diferencias entre las medias. Se consideró para el estudio como significación estadística una $p<0,05$.

Para el estudio se solicitó el consentimiento informado a todos y cada uno de los pacientes antes de empezar la terapia de relajación. Se aseguró la integridad y confidencialidad de todos los datos obtenidos a lo lardo del estudio. La terapia no supuso ningún riesgo para los pacientes.

\section{RESULTADOS}

En el Gráfico 1 se observa que la mayoría de los sujetos del estudio fueron mujeres.

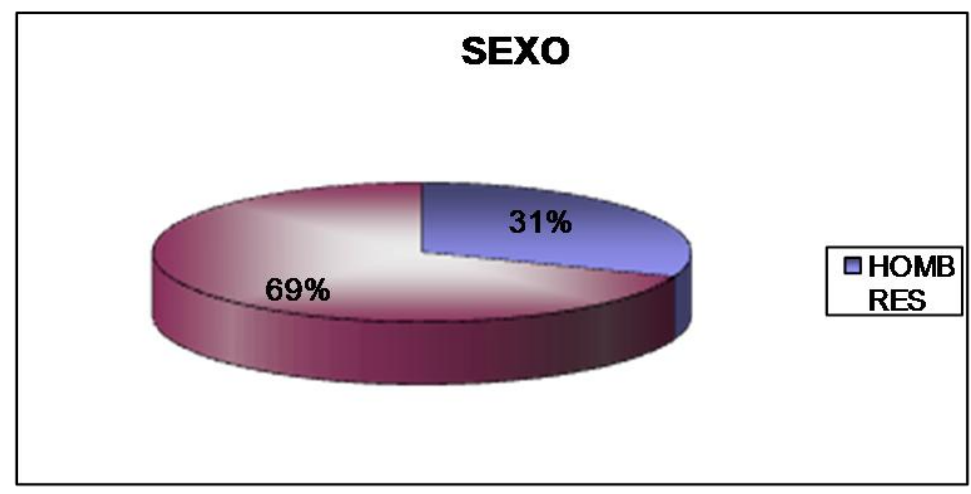

Gráfico 1 


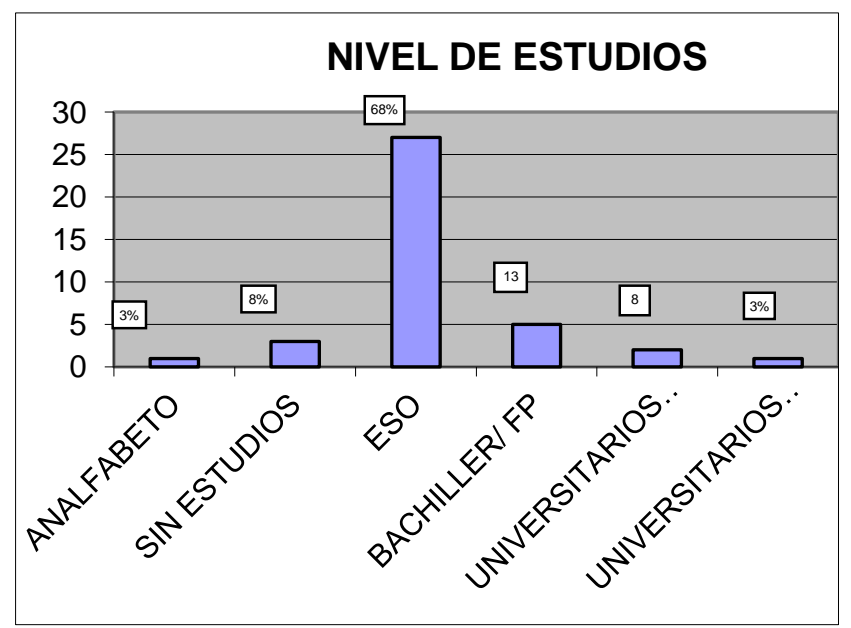

\section{Gráfico 2}

Como se puede observar (Gráfico 2) la mayoría de los sujetos que participaron en el estudio tenía como nivel educativo el grado de educación secundaría obligatoria, en segundo lugar bachiller / formación profesional (FP); en tercer lugar personas sin estudios y universitarios de grado medio y por último analfabetos y universitarios de grado superior.

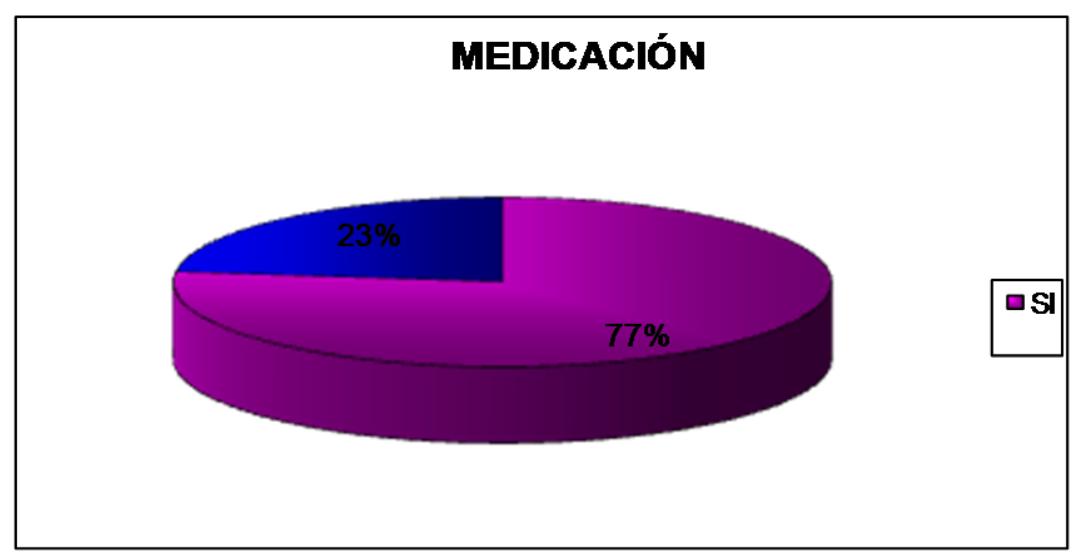

\section{Gráfico 3}

Se observa (gráfico 3) que la mayoría de los sujetos que han participado en el estudio tomaban medicación para disminuir la ansiedad.

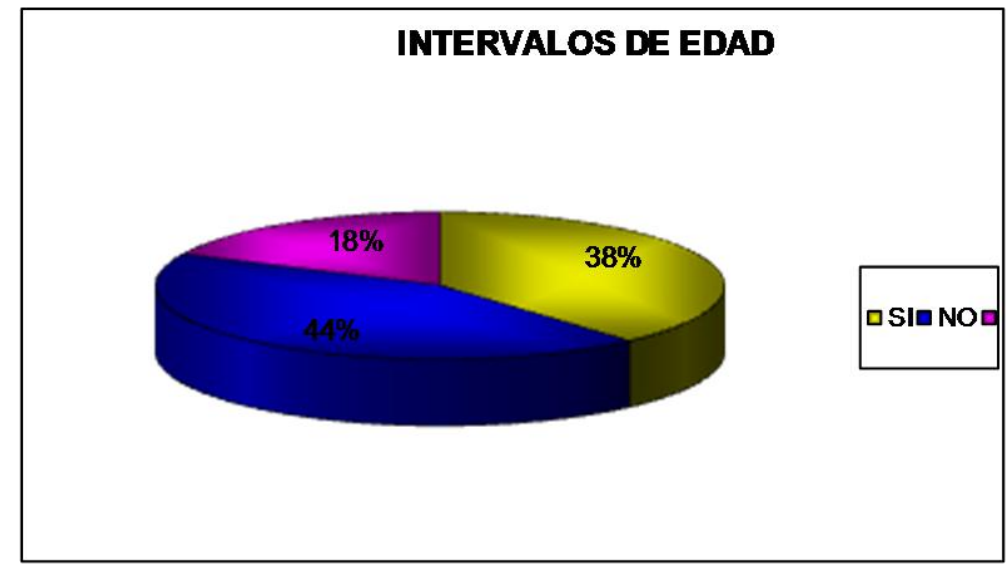

Gráfico 4 
Se observa (gráfico 4 y tabla 1) que el intervalo de edad de 35 a 52 años es el abundante; siendo la media de 39'85 años, la mediana 40 y la moda 33; existiendo un error típico del 1 '88.

\section{Tabla 1}

\section{$E D A D$}

\section{Media \\ Error típico \\ Mediana \\ Moda}

Desviación estándar

Varianza de la muestra

Curtosis

Coeficiente de asimetría

Rango

Mínimo

Máximo

Suma

Cuenta
39,8461538

1,88270718

40

33

11,7575026

138,238866

0,1606518

0,4887496

53

18

71

1554

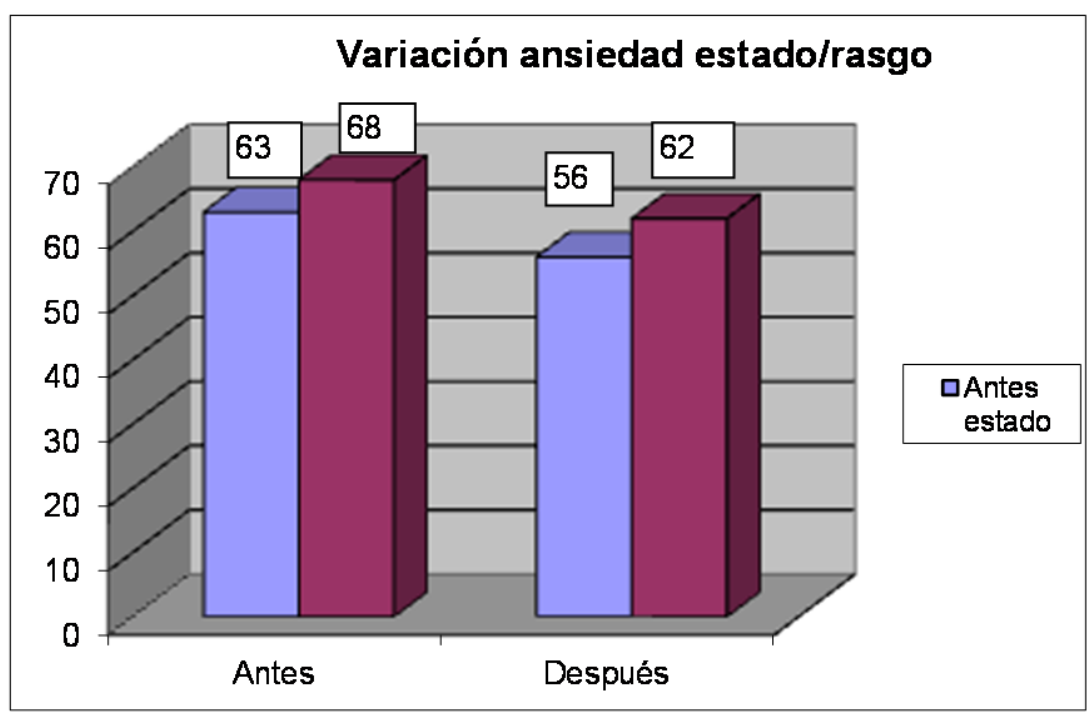

Gráfico 5 


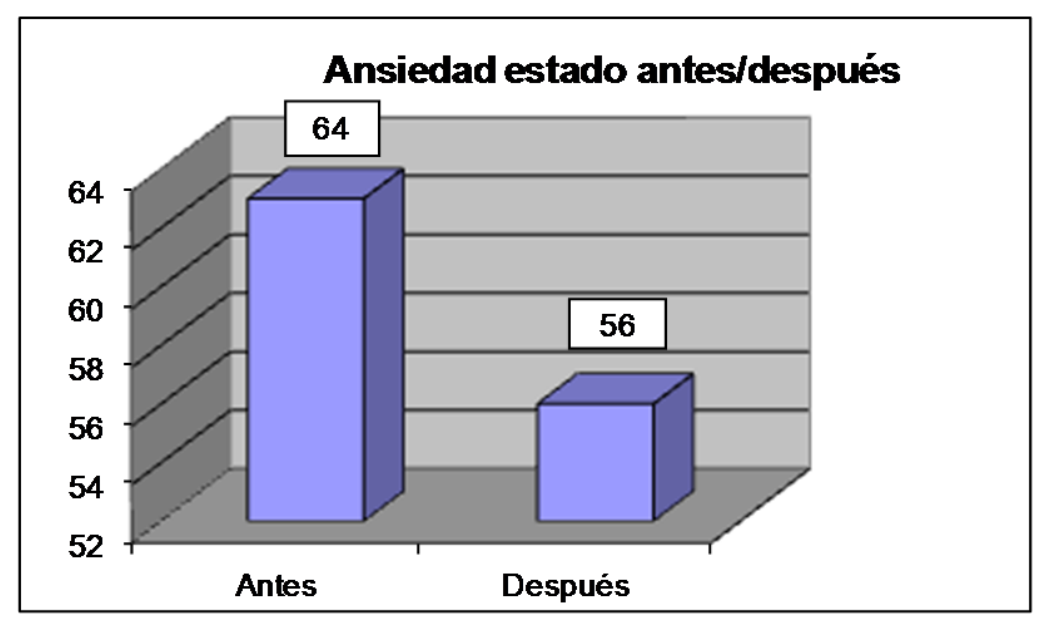

Gráfico 6

Tabla 2

\begin{tabular}{|c|c|c|c|c|}
\hline & Stai antes estado & $\begin{array}{c}\text { Stai después } \\
\text { estado }\end{array}$ & Stai antes rasgo & $\begin{array}{c}\text { Stai después } \\
\text { rasgo }\end{array}$ \\
\hline Media & 68,3589744 & 56 & 68,3589744 & 62,1025641 \\
\hline Error típico & 3,76834016 & 4,21663693 & 3,76834016 & 4,20635001 \\
\hline Mediana & 70 & 50 & 70 & 67 \\
\hline Moda & 65 & 40 & 65 & 70 \\
\hline $\begin{array}{c}\text { Desviación } \\
\text { estandar }\end{array}$ & 23,5332768 & 26,3328892 & 23,5332768 & 26,2686474 \\
\hline $\begin{array}{c}\text { Varianza de la } \\
\text { muestra }\end{array}$ & 553,815115 & 693,421053 & 553,815115 & 690,041835 \\
\hline Curtosis & $-0,18943051$ & $-1,08349861$ & $-0,18943051$ & $-1,15507064$ \\
\hline $\begin{array}{c}\text { Coeficiente de } \\
\text { asimetría }\end{array}$ & $-0,62641212$ & 0,12022085 & $-0,62641212$ & $-0,26719349$ \\
\hline Rango & 89 & 85 & 89 & 83 \\
\hline Mínimo & 10 & 14 & 10 & 16 \\
\hline Máximo & 99 & 99 & 99 & 99 \\
\hline Suma & 2666 & 2184 & 2666 & 2422 \\
\hline Cuenta & 39 & 39 & 39 & 39 \\
\hline
\end{tabular}

Tal y como se aprecia en los gráficos 5,6 y en la tabla 2, se consigue un descenso en el nivel de ansiedad estado desde antes de iniciar la terapia hasta que se finaliza la misma; concretamente el descenso en la puntuación de STAI estado es de una media de 68'35 a 56 puntos, lo cual indica una mejora en el nivel de ansiedad de los pacientes. Sin embargo este descenso no resulta estadísticamente significativo. Al correlacionar las dos medidas de STAI estado (antes/después) se manifiesta una relación entre ellas con un nivel de significación de $p<0$ 027. Sabemos que esa relación es tendente al descenso en la última pero como ya hemos indicado no se ha encontrado la disminución esperada. 


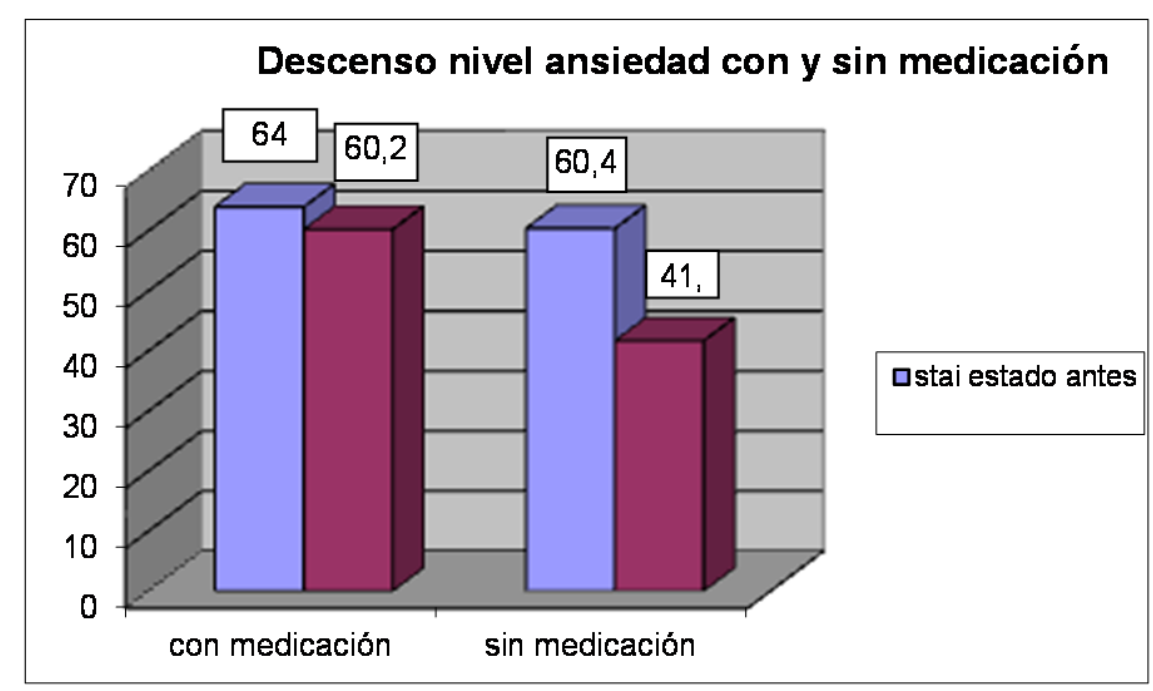

Gráfico 7

Como se observa en el gráfico 7 , existe un claro descenso en el nivel de ansiedad de los pacientes que no llevan medicación al margen de la terapia al margen de un 60,4 a un 41,7; en los pacientes con medicación se puede observar que el descenso es menor.

La comparación de las medidas medias STAI Ansiedad estado antes-STAI ansiedad estado después en los pacientes sin medicación, existen diferencias estadísticamente significativas, al hacer la prueba t de student para muestras relacionadas, con un nivel de $p<0.036$. Por tanto demuestra que las terapias de relajación son eficaces para los pacientes con ansiedad que no tomen medicación y menos eficaces para pacientes sin medición.

\section{DISCUSIÓN}

La población de estudio son pacientes de la zona básica de salud Murcia Infante, que estuvieran diagnosticados de ansiedad y que aceptaran realizar la terapia de relajación.

En el estudio participaron el doble de mujeres que de hombres, de los cuales 30 pacientes tomaban psicofármacos para reducir la ansiedad y 9 no, siendo el nivel de estudios de educación secundaria obligatoria (ESO) el claramente predominante en los pacientes que participaron en el estudio, siendo el intervalo de edad predominante el de 35-52 años. Podemos observar que la ansiedad estado antes-después en pacientes que tomaron medicación para tratar la ansiedad descendió en 4 puntos, mientras que en los pacientes sin medicación descendió unos 19 puntos aproximadamente, por lo que se observa una diferencia significativa entre las dos variables.

Por tanto los resultados indican que hay un descenso del nivel de ansiedad, pero este no es significativo, por lo que se deberá aceptar la hipótesis nula. Respecto a los pacientes con ansiedad que no toman medicación los resultados fueron significativos, de manera que aquí sí se podrá aceptar la hipótesis propuesta.

Burgo Varo ML et al en su estudio: Intervención grupal en los trastornos de ansiedad en atención primaria: técnicas de relajación y cognitivo-conductuales, realizado en el año $2006^{4}$; llegaron a la conclusión de que las terapias de relajación reducían considerablemente los niveles de ansiedad independientemente de si tomaban tratamiento farmacológico o no y sin embargo en este estudio se demuestra que el tomar o no tratamiento farmacológico interviene directamente sobre los efectos positivos de las terapias de relajación. 
Cabello Lázaro $\mathrm{R}$ et al en su estudio: Experimentar la relajación: una técnica para disminuir la ansiedad, realizado en el año $2007^{6}$, llegaron a la conclusión de que la utilización de relajación como terapia no farmacológica de la ansiedad puede realizarse en el ámbito de la atención primaria y especializada, con un profesional de enfermería especialmente preparado

En cuanto a las limitaciones del estudio sin medicación debería haber sido más grande y por separado para ver mejor los efectos. La falta de grupo control supone menor posibilidad de atribuir la mejoría psicológica específicamente a la intervención, ya que también se afectan de tratamiento farmacológico y de actividades de seguimiento en consultas. Por otro lado, el tiempo en que se evalúa el efecto es demasiado próximo a la conclusión de la actividad (generalmente a la semana siguiente) y relativamente próximo a la cumplimentación del test, por lo que pueden intervenir sesgos de recuerdo y de desensibilidad social, además puede existir el sesgo de que los pacientes no respondan sinceramente, por miedo a expresar sentimientos o a lo que pueden pensar los demás.

Podríamos interpretar con los resultados del estudio, que la práctica de terapias de relajación en los centros de salud mental supondría una reducción de ansiedad en pacientes que no estén en tratamiento de psicofármacos para reducir la ansiedad. Por otro lado, sería necesario valorar si añadir a pacientes que estén en terapia con psicofármacos para reducir la ansiedad o no por que el efecto beneficioso que producen es más reducido.

Estos resultados se pueden dar porque los pacientes que toman ansiolíticos ya tienen un reductor de ansiedad y por ello con la relajación no les disminuye tanto, mientras que los que no toman medicación para reducir la ansiedad si disminuye de manera significativa; por lo que sería interesante valorar que quizá no haga falta el uso de psicofármacos, ya que las terapias de relajación disminuyen la ansiedad en pacientes sin tratamiento y en el caso del uso de psicofármacos no complementarla con relajación.

En futuras investigaciones se debe avanzar en el conocimiento del efecto de las terapias de relajación sobre pacientes con ansiedad que tomen tratamiento farmacológico y diferenciarlo de los que no, y valorar si es necesario el uso de ansiolíticos o solo con el uso de la relajación conseguimos efectos deseables. Además se deben abordar investigaciones acerca del efecto de las terapias no farmacológicas. También sería interesante valorar el consumo de psicofármacos.

\section{CONCLUSIONES}

Valorando los resultados del estudio, podemos ver que los talleres de técnicas de relajación pueden ser útiles en la reducción de la ansiedad en atención primaria y especializada, además pueden ser más eficaces en pacientes con ansiedad que no tomen medicación.

Centrándonos en el objetivo primario que era: averiguar si las terapias de relajación benefician a los pacientes con ansiedad; podemos decir que hay una gran diferencia entre los pacientes con medicación y los sin medicación, ya que quizá la medicación haga efecto reductor de la ansiedad y por tanto la terapia ya no reduzca tan significativamente como en el caso de los pacientes sin medicación que al no tener otro aporte reductor de ansiedad, sí que el efecto es muy significativo. Por tanto hay que valorar si con tan solo las terapias de relajación surte efecto y no es necesario dar psicofármacos, evitando así efectos secundarios. 


\section{BIBLIOGRAFÍA}

1. Casañas Sánchez R, Raya Tena A, Ibáñez Pérez L, Valls Colomer MM. Intervención grupal psicoeducativa en pacientes con ansiedad y depresión en atención primaria de Barcelona. El servier \{en linea\} 2009 ffecha acceso 3/11/2009\} 41(4): 229-34. URL disponible en: http://www.elsevier.es/watermark/ctl servlet? $\mathrm{f}=10$ \&pident articulo=13136337\&pident usuar $i \mathrm{io}=0 \&$ pcontactid $=\&$ pident revista $=27 \&$ fichero $=27 \mathrm{v} 41 \mathrm{n} 04 \mathrm{a} 13136337 \mathrm{pdf} 001 . \mathrm{pdf} \& \mathrm{ty}=117 \& \mathrm{acci}$ on=L\&origen=elsevier\&web=www.elsevier.es\&lan=es.

2. Organización Mundial de la Salud. Prevención de los trastornos mentales. Intervenciones efectivas y opciones de políticas. Informe compendiado. Ginebra: OMS; 2004.

3. Garrido Picazo MM. Terapéutica en Atención Primaria: Manejo de la ansiedad en Atención Primaria. El servier \{en linea\} 29-04-2008 \{fecha de acceso 24/11/2009\} 34 (8) 407-10. URL disponible

http://www.elsevier.es/revistas/ctl servlet? $\mathrm{f}=7016$ \&articuloid=13127219\&revistaid $=40$.

4. Burgos Varo ML, Ortiz Fernández MD, Muñoz Cobos F, Vega Gutiérrez P, Bordallo Aragón R. Intervención grupal en los trastornos de ansiedad en Atención Primaria: técnicas de relajación y cognitivo-conductuales. El servier \{en linea\} 2006 \{fecha de acceso 1/11/09\} 32 (5). URL disponible en: http://www.elsevier.es/watermark/ctl servlet? $\mathrm{f}=10$ \&pident articulo=13089378\&pident usuar io $=0$ \&pcontactid $=\&$ pident revista $=40 \&$ fichero $=40 v 32 \mathrm{n} 05 \mathrm{a} 13089378 \mathrm{pdf001}$. pdf \&ty $=12 \&$ accio $\mathrm{n}=\mathrm{L} \&$ origen=elsevier\&web=www.elsevier.es\&lan=es.

5. House A, Stark D. Anxiety in medical patients. BMJ. 2002;325:207-9.

6. Cabello Lázaro R, Brugada Mir M, Aparicio Valero C, Mas MD, Vila Doménech J. Experimentar la relajación: una técnica para disminuir la ansiedad. Rol. 2007; 25 (2): 38- 40.

7.Murphy K. Alivio de la depresión y ansiedad. El servier \{en linea\} 2009 ffecha de acceso 2/12/2009\} $27 \quad$ (2). URL 2 disponible http://www.elsevier.es/revistas/ctl servlet? f=7016\&articuloid=13132974\&revistaid=20.

8. Lunney M, Cavedish R, et all. Diagnósticos enfermeros: Definiciones y clasificación 20102011. Madrid: El Sevier; 2010.

9. Martín R, Buenestado C, Caballero ML, Lora F. Aten Primaria. 2000; 25: 518-9.

10. Echeburúa E. Evaluación y tratamiento el trastorno por ansiedad generalizada: nuevas perspectivas. Psicología Conductual; 1993: 233-254.

11. American Psychiatric Association. Manual diagnóstico y estadístico de los trastornos mentales: Atención Primaria. DSM-IV-TR. AP. Masson. Barcelona. 2004.

12. Pacheco Yánez L, Medrano Albéniz J. Psicofarmacología, aplicada en atención primaria. Barcelona: Glosa; 2007.

13. Aragüés M, Fernández Esteban I, Jiménez Arriero MA, et al. Recomendaciones para el manejo de los trastornos depresivos y de ansiedad en Atención Primaria. Recomendaciones farmacoterapéuticas en salud mental. Revisión 2010. Dirección General de Farmacia y Productos Sanitarios. Consejería de Sanidad y Consumo. Comunidad de Madrid. Disponible en http: //www.madrid.org/sanidad/farmacia/recomendaciones.

14. Carbelo Baquero B, Casas Martínez MF, Romero Llort M, Rosique Robles A. Relajación: habilidad con la práctica. Enferm. Cientif. 2007; 32 (35): 188-89.

15. García López J, Torre Peña M J, Sánchez Jurado L I, Jiménez Díaz M C, Lupiáñez López Y. Desarrollo del modelo de autocuidados de Dorotea Orem en paciente en D.P.C.A. Rev Soc Esp Enferm. 1998. 3;15-21.

16.Gómez Pesquera C, Francisco del Rey C, Sanz Criado C. Estudio de un caso desde la perspectiva de Dorotea Orem. Educare21. 2004. 
17. Memoria Paiva L, Venicios de Oliveira M, Augusta Batista V. Componentes funcionales de la teoría de Peplau y su confluencia con el referencial de grupo. Acta enferm. 2006; 19 (2): 228-233.

18. Bravo Martín R. Optimizando el entrenamiento en relajación. El servier \{en linea\} 2005 \{fecha de acceso 4/12/2009\} 3 (2). URL disponible en: http://www.ee.enfermundi.com/servlet/Satellite?pagename=ExcelenciaEnfermera/Articulo E E/plantilla articulo EE\&numRevista $=3 \&$ idArticulo $=1103724904810$.

19. Vilar Hernández M, Cano Carrascosa J. Taller de autoayuda y ansiedad de enfermería. \{en linea\} 2009 fecha de acceso 14/12/2009\} URL disponible en: http://www.codem.es/Docs/Noticias/taller de auto ayuda.pdf.

20. Aguilera Manrique G, Belmonte García MT, Zapata Boluda R, Granados Gámez G, Marcos Perez E, Cristóbal Cañadas C. Procedimientos de relajación. Metas. 2003 ; 5 (51): 14-18.

21. Illan I, Hurtado A, Marin JL. Protocolo de relajación. Murcia: Servcio Murciano de Salud; 2004.

22. Luebbert K, Dahme B, Hasenbring M. The effectiveness of relaxation training in reducing treatment-related symtoms and improving emotional adjustment in acute non-surgical cancer treatment: a meta-analytical review. Psychooncology. 2001 Nov-Dec; 10 (6):490-502.

23. Hyman RB, Feidman HR, Harris RB, Levin RF, Mallory GB. The effectiveness of relaxation training on clinical symptoms: a meta-analysis. Nurs Res. 1989 Jul-Aug; 38 (4): 216-20.

24. Stetter F, Kupper S. Autogenic training: a meta-analysis of clinical autcome studies. Appl Psychophysiol Biofeedback. 2002 Mar; 27 (1): 45-98.

25. Rood YR, Bogaards M, Goulmy E, Houwelingen HC. The effects of stess and relaxation on the in vitro inmune response in man: a meta-analytic study. J. Behav Med. 1993 Apr; 16 (2): 163-81.

26. Sánchez Meca J, Rosa Alcázar Al, Olivares Rodríguez J. Eficacia diferencial de los tipos de relajación en problemas de salud: un estudio meta-analítico en España. Cuaderno de Medicina Psicosomática. 1998; 45-46, 21-36.

ISSN 1695-6141

(๖) COPYRIGHT Servicio de Publicaciones - Universidad de Murcia 